\title{
Convergent Adaptation in Mitochondria of Phylogenetically Distant Birds: Does it Exist?
}

\author{
Valentina Burskaia $\mathbb{D}^{1,2, *}$, llja Artyushin ${ }^{3}$, Nadezhda A. Potapova ${ }^{2}$, Kirill Konovalov ${ }^{4}$, and Georgii A. Bazykin ${ }^{1,2}$ \\ ${ }^{1}$ Center of Life Sciences, Skolkovo Institute of Science and Technology, Moscow, Moscow Oblast, Russia \\ ${ }^{2}$ Molecular Evolution Laboratory, Institute for Information Transmission Problems of the Russian Academy of Sciences (Kharkevitch Institute), \\ Moscow, Russia \\ ${ }^{3}$ Department of Vertebrate Zoology, Faculty of Biology, Lomonosov Moscow State University, Moscow, Russia \\ ${ }^{4}$ Department of Chemistry, Hong Kong University of Science and Technology, Kowloon, Hong Kong \\ *Corresponding author: E-mail: valentina.burskaia@skolkovotech.ru.
}

Accepted: 15 May 2021

\begin{abstract}
In a wide range of taxa, proteins encoded by mitochondrial genomes are involved in adaptation to lifestyle that requires oxygen starvation or elevation of metabolism rate. It remains poorly understood to what extent adaptation to similar conditions is associated with parallel changes in these proteins. We search for a genetic signal of parallel or convergent evolution in recurrent molecular adaptation to high altitude, migration, diving, wintering, unusual flight abilities, or loss of flight in mitochondrial genomes of birds. Developing on previous work, we design an approach for the detection of recurrent coincident changes in genotype and phenotype, indicative of an association between the two. We describe a number of candidate sites involved in recurrent adaptation in ND genes. However, we find that the majority of convergence events can be explained by random coincidences without invoking adaptation.
\end{abstract}

Key words: convergence, GWAS, mitochondria, birds.

\section{Significance}

Previous attempts to detect convergent single-nucleotide mutations in phylogenetically distant species were often limited by a few number of convergent phenotype acquisitions. We design a data set in which similar traits appear independently dozens of times. We show that convergent single-nucleotide mutations are still unlikely in phylogenetically distant species.

\section{Introduction}

Mitochondrial genes were repeatedly claimed to adapt in response to lifestyles that require oxygen starvation or elevation of metabolism rate in a wide range of eukaryotic taxa (Das 2006). The most obvious candidate species for the evolution of hypoxia tolerance are those inhabiting high altitudes. Indeed, high-altitude adaptations have been described for mitochondrial genes of many species, including the COX3 gene of the bar-headed goose (Scott 2011), ATP6 and ATP8 in shrimps from genus Artemia (Zhang 2013), ND5 in caterpillars of genus Gynaephora (Yuan et al. 2018), COX1 in Tibetan antelope Pantholops hodgsonii (Xu et al. 2005), and ND2, ND4, and ATP6 in Tibetan galliform birds (Zhou 2014). Adaptations to high altitude in human populations typically involve mutations in ND1 as well as probably other genes (Ji et al. 2012; Kang et al. 2013). Besides high-altitude hypoxia, some rodents likely developed adaptations to subterranean hypoxia (Tomasco and Lessa 2011).

Life at extreme temperatures and extraordinary physical activity could also cause adaptive evolution in mitochondrial genes through their effect on energy metabolism. It has been shown that genes involved in oxidative phosphorylation

(C) The Author(s) 2021. Published by Oxford University Press on behalf of the Society for Molecular Biology and Evolution.

This is an Open Access article distributed under the terms of the Creative Commons Attribution License (http://creativecommons.org/licenses/by/4.0/), which permits unrestricted reuse, distribution, and reproduction in any medium, provided the original work is properly cited. 
(OXPHOS) could take part in adaptation to the arctic environment: adaptations in ND1, ND3, and ND4 genes were described in the Atlantic salmon (Consuegra et al. 2015), and adaptations in CYTB gene were found in European anchovy (Silva et al. 2014). Furthermore, there is evidence for adaptation to long-range migrations in the yellow-rumped warbler (Toews 2014). Flight is another energy-consuming adaptation, and some studies confirm adaptation of the mitochondrial genes to flight in bats (Shen et al. 2010).

Most evidence for selection in mitochondria is indirect. It often comes down to description of differences in frequencies of a few alleles, which could be a consequence of random drift in small populations. Positions discovered in different studies rarely overlap, suggesting that different organisms use different mechanisms of OXPHOS system adaptation to similar environments, or that some of the findings are erroneous. Still, some gene regions are more likely to be affected by adaptation (da Fonseca et al. 2008). Furthermore, the role of the mitochondrial electron transport chain in physiological acclimation was demonstrated in many experimental studies. For example, gene expression, protein abundance, and the enzyme activity changes in plants and animals in process of cold acclimation (Lucassen 2006; Armstrong et al. 2008).

To study this systematically, we decided to conduct a broad search for adaptive convergent evolution in mitochondria of birds in an attempt to find universal genotype-to-phenotype associations. We concentrated on mitochondrial adaptations in bird species that likely face hypoxia (high altitude and diving) or requirement for elevated (long-distance migration, wintering at high latitudes and unusual flight abilities) or reduced (loss of flight) rate of metabolism, hypothesizing that detected adaptations may confer resistance to these types of physiology.

To estimate potential convergence, we develop upon an existing phylogenetic test for detection of parallel adaptation (TreeWAS package, Collins and Didelot 2018). This test is based on reconstruction of ancestral states in the internal nodes of the phylogeny, and then counting the number of coincident changes of phenotype and genotype at each amino acid site at the same branch of the phylogenetic tree. Additionally, at each site, we measure the change of amino acid propensities associated with phenotype change (PCOC package, Rey et al. 2018). In total, we analyzed 415 species. While we detect a number of candidate sites that could be associated with convergent adaptation to high altitude and long-distance migration in birds, we only detect one site that was associated according to both tests, and this association was borderline significant. Overall, we find little or no signal of recurrent adaptation, indicating that adaptation to extreme physiology in birds can proceed via different routes in different species, and/or that it can be largely driven by nonmitochondrially encoded genes.

\section{Results}

Simultaneous Change

All three types of simultaneous score metrics revealed that the number of significant associations was low. The highest number of sites (8) was detected in high altitude birds, all of them of marginal significance. The Convergence test detected two sites in ND1 and ND5 genes (fig. 1). The GWAS test detected another two sites in the ND2 gene (supplementary fig. S1, Supplementary Material online). The All changes test detected six sites in ND1, ND2, ND4, ND5, and ND6 genes (supplementary fig. S3, Supplementary Material online). Findings of different tests partially overlap (table 1). Additionally, the All changes test detected the site associated with adaptation to long-distance migration in the ND5 gene. When the stronger Bonferroni correction was applied, only two sites detected by the Convergence test in high altitude birds and one site detected by the All changes test in long-distance migrants remained significant (table 1).

\section{Profile Change}

As an additional test for convergence, we use the amino acid Profile change metric. We expect that recurrent mutations emerging simultaneously with convergent phenotype change could also lead to a change in the amino acid profile between the branches carrying the foreground and the background phenotypes. We compared the results of the Simultaneous tests under all three approaches with the Profile change test (fig. 2, supplementary figs. S2 and S4, Supplementary Material online).

First, we were interested to estimate profile change levels at sites that have a significant Simultaneous score. Among them, only one (57th position of ND5, detected by the Convergence test) has a profile change score above 0.5 (0.82) and thus can be considered as potentially convergent. Others either result from convergent changes to the same amino acid without a profile change (17th position of ND1), or are a consequence of divergent evolution (all other positions).

Second, we could expect that sites with higher simultaneous score could have higher profile change metric if a substantial fraction of these sites is involved in convergent adaptations. To test this assumption, we arbitrarily divided the plots (fig. 2, supplementary figs. S2 and S4, Supplementary Material online) into four parts, and tested if sites with higher simultaneous score have higher profile change score. Profile change scales were simply divided into two parts by the middle. Simultaneous score scales are discrete with small number of values, thus the cutoff threshold was selected manually around the middle of the scale. We found no dependency in any of the tested phenotype groups (Fisher test, significance level 0.01). Particularly, excess of points in the upper right corner of each graph, which could be formed by nonrandom highly convergent sites, was not detected. 

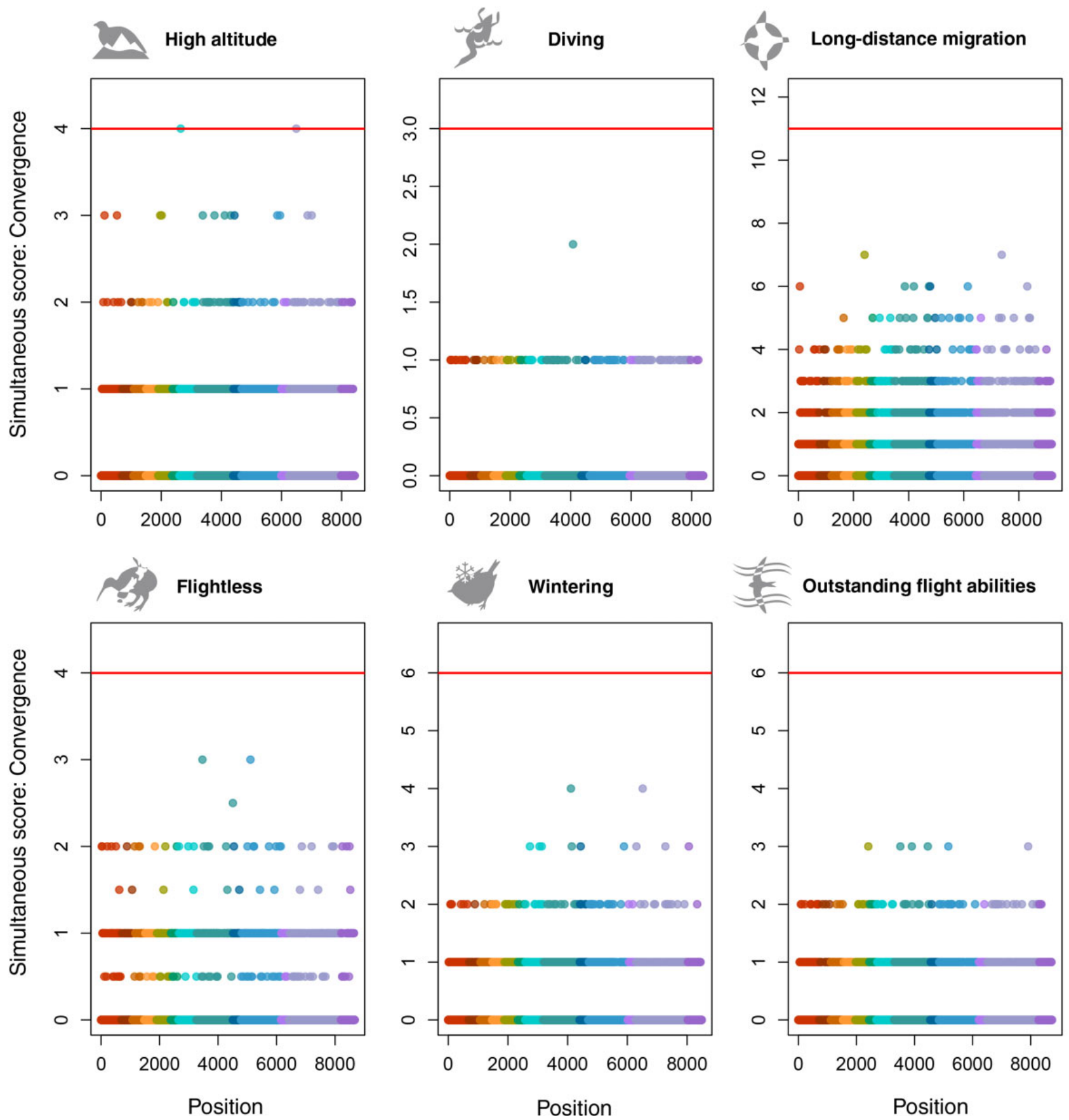

сутв

ATP6

ATP8

ND1 ND2

ND3

ND4

ND4L ND5

ND6

FIG. 1.-Simultaneous test based on the Convergence approach for each of the six considered phenotypic traits. Horizontal axis, position in the mitochondrial genes; vertical axis, number of simultaneous changes of phenotype and genotype. Red line corresponds to significance threshold 0.05 with Bonferroni correction accounting for the number of considered sites in particular test and phenotype (Correction 1).

\section{Sites with Evidence for Phenotypic Association}

Position 57 in the ND5 gene carries the highest signal of functional convergence, as both the Simultaneous and Profile change scores are rather high for it (4 and 0.82 , respectively). However, except for the four substitutions from Histidine to
Tyrosine that occurred synchronously with the phenotype change, there are tens of other substitutions between these two amino acids that occurred at various positions of the phylogeny (partially shown in fig. 3). This suggests that this convergence can be accidental. 
Table 1

List of Significant Substitutions Detected by the Simultaneous Test

\begin{tabular}{|c|c|c|c|c|}
\hline \multirow{2}{*}{$\begin{array}{l}\text { Test Type } \\
\text { High altitude }\end{array}$} & \multicolumn{4}{|c|}{ Gene Position in Gallus gallus Correction 1 Correction 2} \\
\hline & & & & \\
\hline \multirow[t]{2}{*}{ Convergence } & ND1 & $17 a a(I)$ & + & + \\
\hline & ND5 & $57 a a(H)$ & + & + \\
\hline \multirow[t]{6}{*}{ All changes } & ND1 & $17 a a(I)$ & + & - \\
\hline & ND2 & $329 a a(T)$ & + & - \\
\hline & ND4 & $418(\mathrm{~T})$ & + & - \\
\hline & ND5 & $114 a a(F)$ & + & - \\
\hline & ND5 & $495 \mathrm{aa}(\mathrm{T})$ & + & - \\
\hline & ND6 & $135(V)$ & + & - \\
\hline \multirow[t]{2}{*}{ GWAS } & ND2 & 278(M) & + & - \\
\hline & ND2 & 329aa(T) & + & - \\
\hline \multicolumn{5}{|c|}{ Long-distance migration } \\
\hline All changes & ND5 & $533(\mathrm{~T})$ & + & + \\
\hline
\end{tabular}

Note.-Correction 1 column indicates the sites that remain significant after Bonferroni correction accounting for the number of considered sites. Correction 2 column indicates the sites that remain significant after Bonferroni correction accounting for the number of sites and the number of tests ( 3 tests: Convergence, All changes, and GWAS).

Other positions with relatively high Simultaneous scores demonstrate low Profile change scores. Thus, at best some of these sites could be involved in divergent evolution associated with phenotype changes. We used the MEME tool (Kosakovsky Pond et al. 2005) on ND genes to find sites involved in the recurrent positive selection, yet there was no overlap with our findings.

As all nine candidate positions were in ND genes, we suggest that adaptations could be associated with the respiratory complex I. Among all 3,602 analyzed amino acid positions, ND genes account for 1,998 positions (55\%), so it is unlikely to be a coincidence. To ask if there is additional evidence for function, we mapped the candidate positions onto the 3D structure of the respiratory complex I (fig. 4). All the positions are far from the FeS electron-transport clusters and are buried into the membrane arm of the respiratory complex I. They are not grouped together, and they are not close to polar residues in proton channels that play a key role in proton transport (Fiedorczuk 2016). In total, structural data provide no additional evidence to consider these sites as adaptive.

\section{Alternative Approach: PCOC}

Hypervariable sites may be the main weakness of TreeWAS because it does not account for the background rate of change for a given amino acid position. In an attempt to verify our results, we applied PCOC (Rey et al. 2018)—a method for convergent evolution detection, which is sensitive to site evolutionary rate heterogeneity (Supplementary Material online). The method detected two convergent sites: 278th position in ND2 gene is associated with loss of flight and 17th position in ND5 gene is associated with diving. Remarkably, 278th position in ND2 gene was previously discovered by TreeWAS as associated with high altitude adaptation.

However, like the nine candidate sites, discovered by TreeWAS, these sites are far from active centers of the respiratory complex I (supplementary fig. S6, Supplementary Material online). Each of these sites undergoes tens of mutations, not associated with phenotype change (supplementary figs. S7 and S8, Supplementary Material online). It leads us to the assumption that these positions are also hypervariable.

\section{Discussion}

The site with the strongest signal of convergence detected in our analysis, which has high Convergence and Profile change scores (position 57 in ND6), could originate from functional convergence. Alternatively, the observed pattern of changes could still be coincident, as the mutation pattern rather looks like switches between the set of permitted amino acid under time-invariant amino acid constraints. Among other, basically divergent, positions, none provided additional evidence for selection. We suppose that the concentration of significant associated substitutions in the ND genes could be a consequence of higher mutation rates in ND genes.

Though we detect no significant associations at the amino acid level, we hypothesized that sites with higher Simultaneous scores could have elevated Profile change scores. This could be the case if instead of a few strong associations, the data carried sites with convergent associations at a small group of phylogenetically close species, or if the associations were weak. However, we detect no excess Profile change score among the sites with higher Simultaneous scores.

The application of an alternative approach for convergent evolution detection (PCOC), which accounts for background rate of change for a given amino acid position, was not helpful. Two significant sites were found, although both are high variable and are remote from protein active centers (minimal distance $11.3 \AA$ ) , which makes these findings doubtful.

Previous works have attempted to detect convergent single-nucleotide mutations in such distant groups as marine mammals (Foote et al. 2015) or echolocating bats and whales (Parker et al. 2013). Many of these attempts failed to find significant convergence or were disproved by later studies (Thomas and Hahn 2015; Zou and Zhang 2015). Similar to those works, we here explore the convergence between distantly related species. All the phenotypes analyzed here were acquired repeatedly (table 2), supposedly making the convergence-based analysis of adaptation more powerful. However, our results did not support this assumption. This suggests that adaptive convergent evolution is rare or hardly detectable in bird OXPHOS system at the considered phylogenetic distances.

There remains a possibility that convergent adaptations in the OXPHOS system could be found in groups of close 


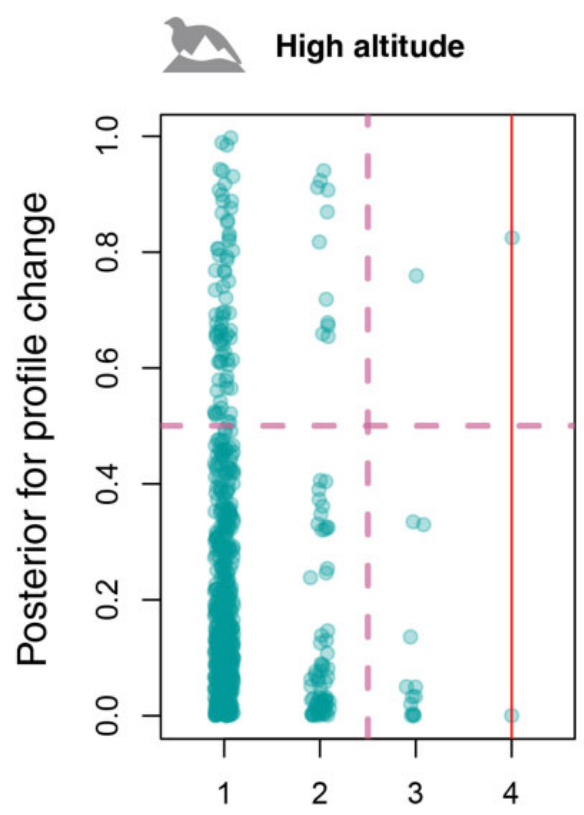

Flightless

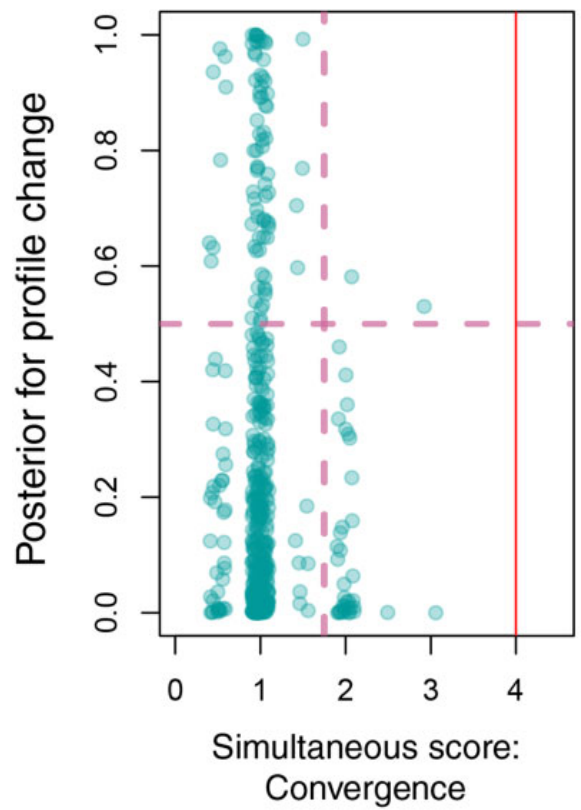

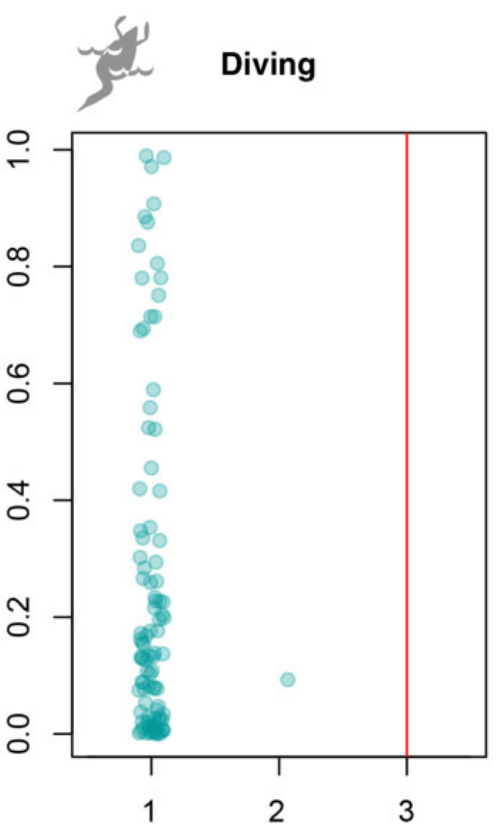
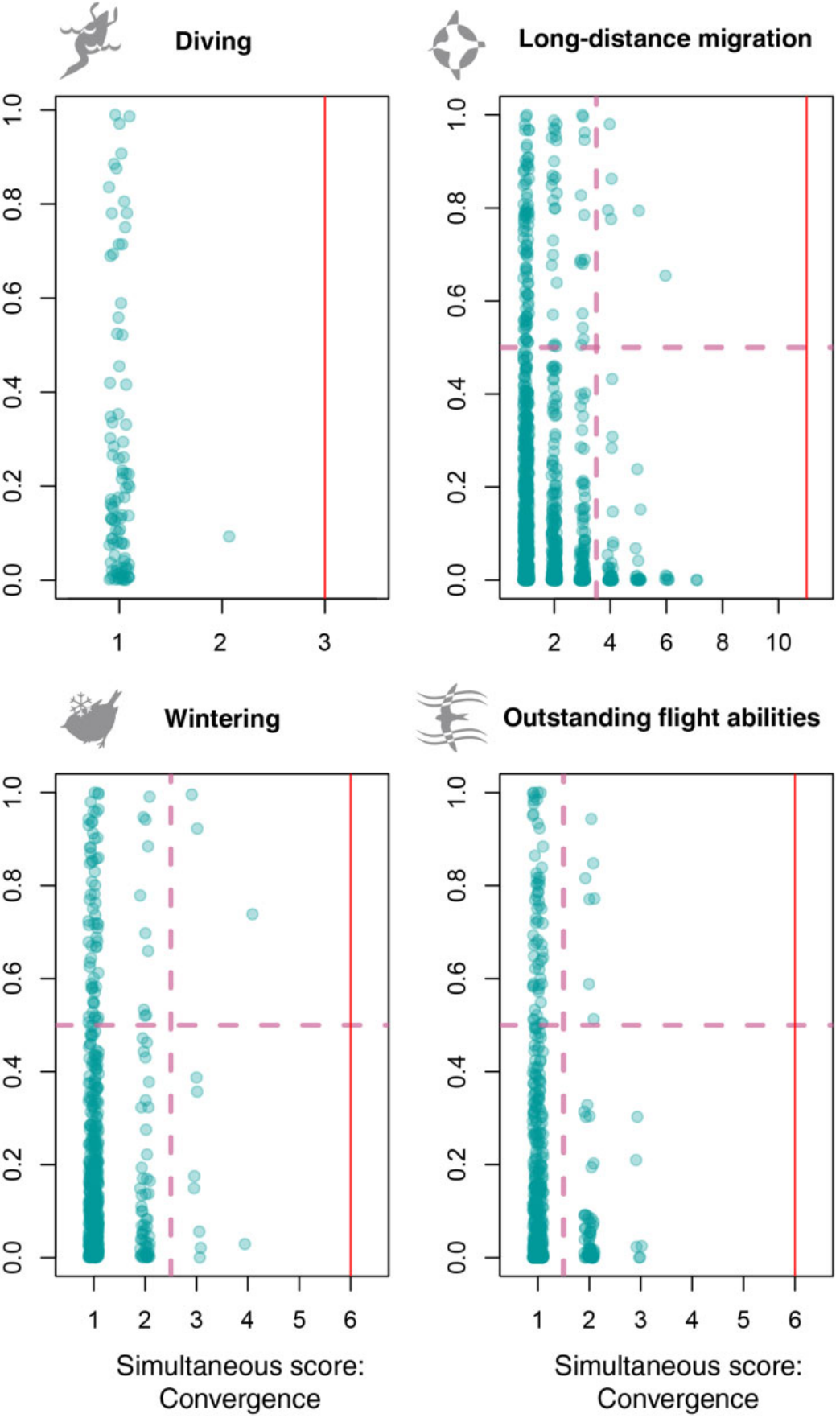

Fig. 2.-Profile change versus Simultaneous score (Convergence approach). The red line shows the significance threshold for the Simultaneous test. The dashed lines show division of the plot for the Fisher test.

relatives when the tree of life will be sequenced with higher density. As it was shown by Natarajan et al. (2016), similar mutations in hemoglobin subunits lead to high latitude adaptations only in a similar genetic context: there are typical "hummingbird high altitude mutations" and typical "duck high altitude mutations." If so, the near-lack of signal in our study has to do with the fact that it was based on too distant organisms which have highly divergent evolutionary landscapes in the genes of interest. However, other work indicates that similar substitutions are rarely involved in independent adaptation to high altitudes even inside a group of closely related species like hummingbirds (Lim et al. 2019).

Further work may improve the search for simultaneous changes by using better statistical models. Specifically, these 

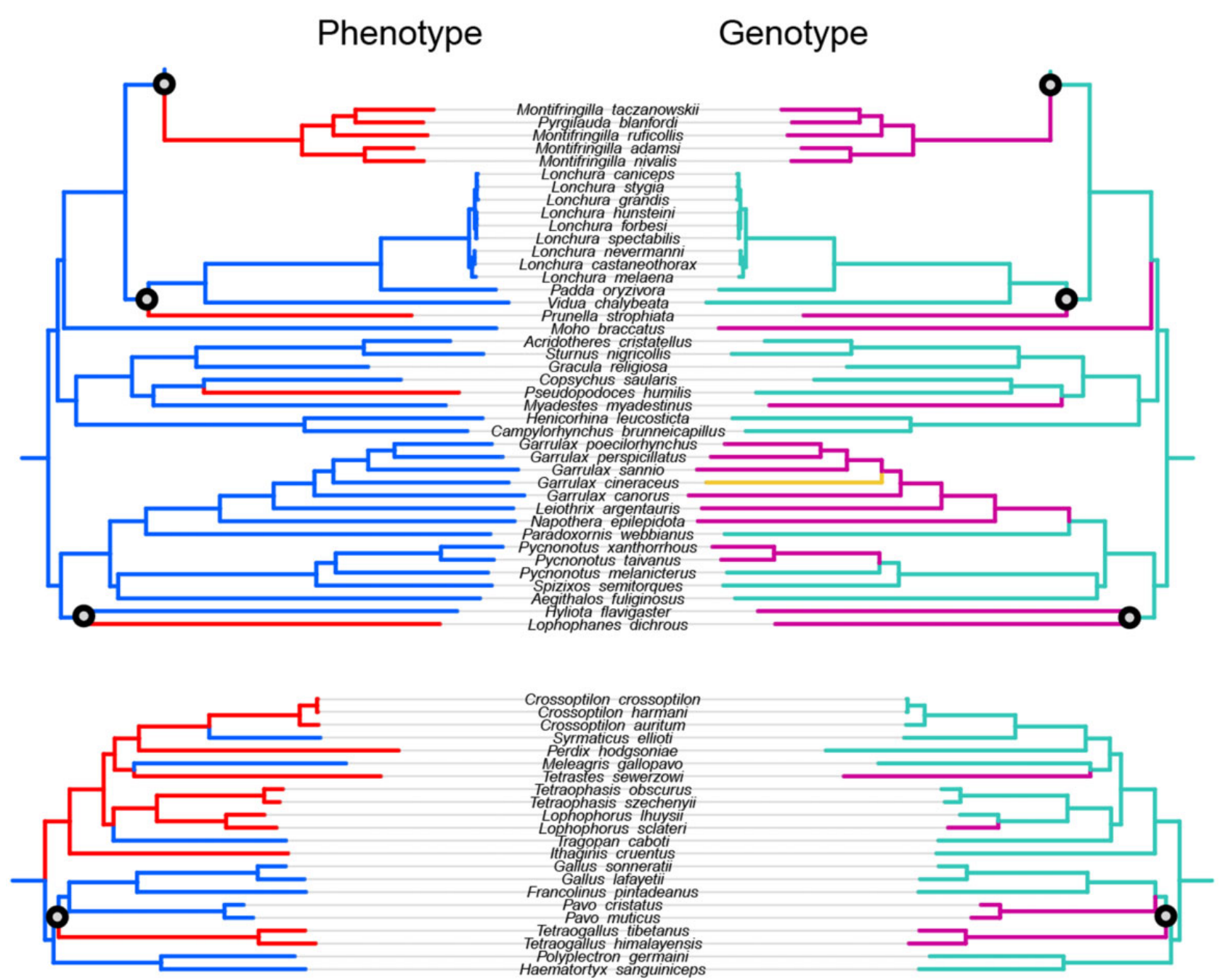

Background High altitude

Histidine Tyrosine $\quad$ Serine

FIG. 3.-Position 57 in the ND5 gene associated with high altitude adaptation. This position carries 4 substitutions from Histidine to Tyrosine simultaneous with adaptation, and a Profile change score of 0.82 . Only branches with simultaneous changes are displayed.

models could be improved by incorporating better processing of the heterogeneity of the substitution rates between sites or phylogenetic branches.

\section{Materials and Methods}

Phenotypes

We analyzed 415 species of birds. All species were divided into seven groups according to their phenotypic characteristics. Phenotypes were classified in accordance with the Bird of the World research database (https://birdsoftheworld.org, accessed August 20, 2020). As high altitude, we classified those species for which the lower boundary of the range was over $2,000 \mathrm{~m}$, and the upper boundary of the range, over 4,000 $\mathrm{m}$ above sea level. As divers, we classified those species which can spend at least several minutes underwater. As species with the ability for long-distance migration, we considered those species with nonoverlapping or weakly overlapping breeding and wintering ranges. As wintering, we classified those species which are typically exposed to subzero temperatures and snow cover for many months each year. We also formed two samples of species with specific flightrelated phenotypes: flightless birds, a phenotype which has originated repeatedly in different groups of island birds; and birds with outstanding flight abilities. The latter group united swifts, hummingbirds, swallows, terns and gulls, scuas, gannets, tropicbirds, falcons, and accipiters. Although the similarity of these adaptations may be controversial, we hypothesize that the lifestyles of all these groups involve high energy demand and thus could affect the mitochondrial genes similarly. 


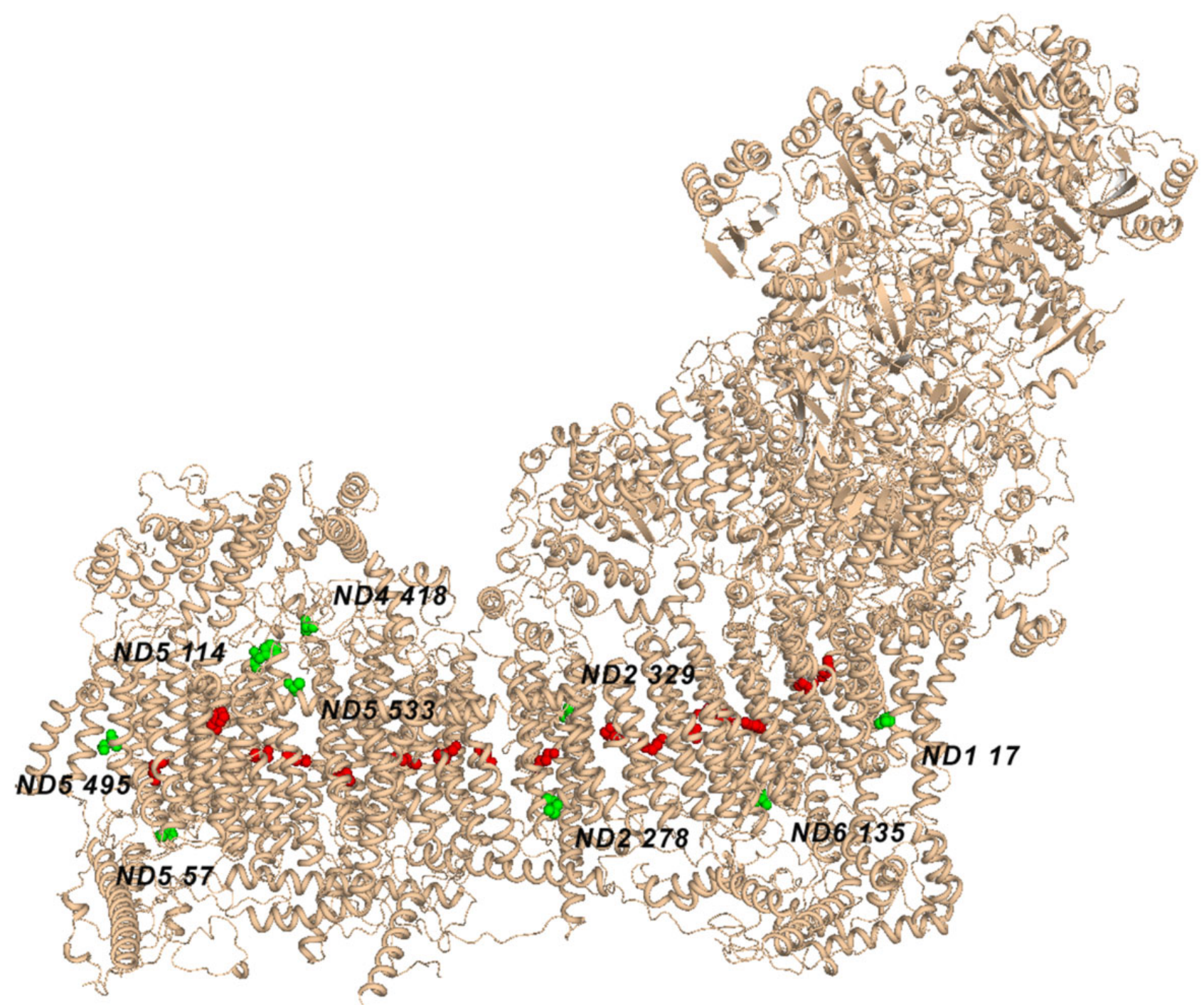

FIG. 4.-3D structure of respiratory complex I. Candidate amino acid residues are colored green. Polar residues in proton channels, which play a key role in proton transport, are colored red.

Table 2

Number of Species in Each Phenotypic Group

\begin{tabular}{lcc}
\hline & Number of Species & $\begin{array}{c}\text { Number of Times the Phenotype Emerged } \\
\text { Independently }\end{array}$ \\
\hline High altitude & 23 & 7 \\
Diving & 25 & 3 \\
Long-distance migration & 91 & 33 \\
Wintering & 28 & 11 \\
Flightless & 33 & 6 \\
Outstanding flight abilities & 58 & 7 \\
Reference & 174 & - \\
\hline
\end{tabular}

To study phenotypic associations, we also need a reference group of species which do not carry the specific adaptations considered in this work. The choice of such a reference is a complicated task, because of the complexity of natural ecological adaptations. As the reference group, we decided to use tropical and subtropical birds with ranges not extending 
A

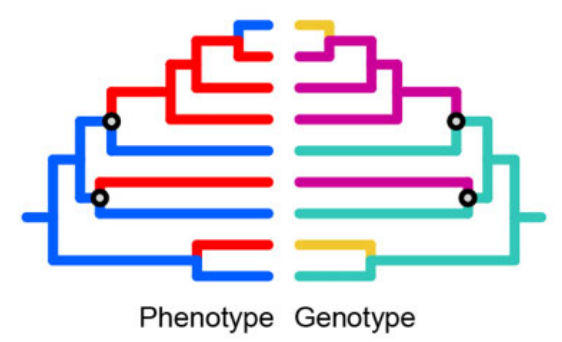

B

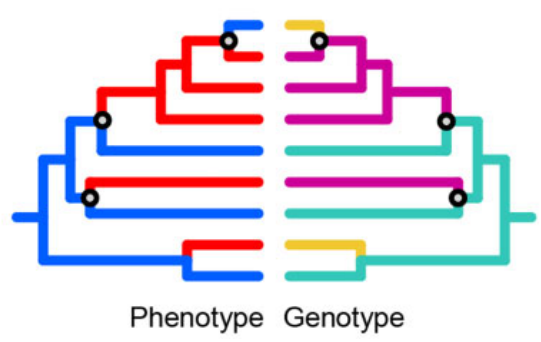

C

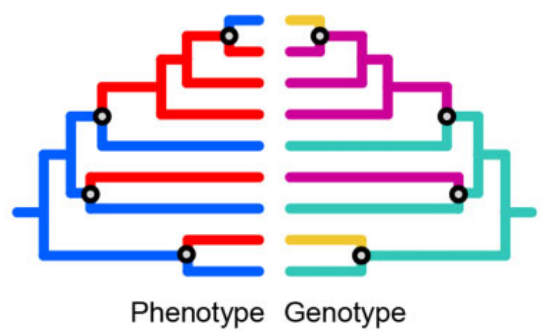

FiG. 5.-Three approaches to counting the number of simultaneous changes in the phenotype and the encoded amino acid. In each panel, the two trees facing each other show the same phylogeny, with the coloring corresponding to the trait states in the corresponding branches; phenotype in the left, and amino acid at a particular position in the right. Circles indicate changes in phenotype and genotype deemed coincident under the corresponding approach. (A) Under the Convergence approach, of the three gains of the foreground phenotypic trait state (blue to red), two coincide with the gains of the foreground amino acid (cyan to purple). (B) Under the GWAS approach, two gains (blue to red) and one loss (red to blue) of the foreground phenotypic trait state coincide respectively with two gains (cyan to purple) and one loss (purple to yellow) of the foreground amino acid. (C) Under the All changes approach, four changes in the phenotype coincide with four changes in the encoded amino acid. The corresponding numbers of simultaneous events is two (A), three (B), and four (C).

above 2,500 $\mathrm{m}$ and which have none of the specific aforementioned adaptations. The number of species in each phenotypic group is provided in table 2 , and the list of species is provided in supplementary fig. S5, Supplementary Material online.

\section{Gene Sequences and Phylogeny}

We downloaded complete mitochondrial sequences of birds from Genbank (https://www.ncbi.nlm.nih.gov/genbank/, accessed August 20, 2020). The sequence of each of the 13 genes was obtained according to the GenBank annotation. Species with duplicated genes were excluded from the analysis. Sequences of each gene were aligned independently with MACSE toolkit (Ranwez et al. 2011). Columns of alignment with gaps were excluded by trimAl software (CapellaGutierrez et al. 2009). Phylogenetic tree reconstruction was based on concatenated nucleotide alignments of 13 genes. It was performed in IQ-Tree 2 package (Minh et al. 2020), the alignment was split into 39 partitions by gene and codon position. As early divergences in the bird tree of life are discordant (Jarvis et al. 2014), we used constraints for bird orders branching in our reconstruction. We applied constraints from the most recent revision of bird phylogeny (Kimball 2019), which combines nuclear and mitochondrial data to construct a consensus supertree for 707 bird species. Amino acid ancestor states reconstruction was also performed in IQ-Tree with the usage of genewise partitioned mtVer evolutionary model.

\section{Search for Convergent Evolution and Phenotype to Genotype Associations}

To count simultaneous changes of phenotype and genotype at the site, we use the simultaneous score of the TreeWAS package (Collins and Didelot 2018). The simultaneous score is designed for the so-called phylogenetic GWAS analysis. It splits each alignment column into binary (two-state) SNPs and counts simultaneous changes of phenotype and genotype at tree branches. To estimate the probability that the observed association is nonrandom, TreeWAS simulates a "null" genetic data set under the empirical phylogenetic tree and terminal phenotypes. It also takes from empirical data the distribution of numbers of substitutions per site. In each simulation, it counts the number of phylogenetic branches with simultaneous changes of phenotype and genotype and combines these counts to obtain the null distribution. At the upper tail of the null distribution, a threshold of significance is drawn at the quantile corresponding to [1 (alpha-level corrected for multiple testing)]. If a site in the real data set has more simultaneous changes than the threshold, it is considered to be significantly associated with the corresponding phenotype.

TreeWAS was originally developed for the analysis of whole-genome data sets of closely related species, in which the assumption that no more than two variants may occur in any particular site generally holds. By contrast, in distantly related bird species considered here, the amino acid sequence of mitochondria has frequently undergone multiple substitutions per site. Therefore, we had to adapt TreeWAS for dealing with nonbinary SNPs. This is nontrivial, as we have no a priori knowledge which of the amino acid changes may be associated with changes in the phenotype.

We used three approaches to identify substitutions that coincide with changes in phenotype. Generally, each approach resulted in different sets of branches on which convergent substitutions occurred, and therefore different estimates of simultaneous score.

In the first and in the second approach, we designated one amino acid as foreground and did not distinguish between the remaining amino acids. We also designated one 
phenotype (of the two possible phenotypes) as foreground. In the first approach, as genotype changes, we only considered the gains of the foreground amino acid; and as phenotype changes, we only considered the acquisition of the foreground phenotype. As coincident changes, we considered the phylogenetic branches where these events coincided. This approach is referred to as "Convergence" (fig. 5A).

In the second approach, as genotype changes, we considered both the gains and the losses of the foreground amino acid, and as phenotype changes, both the gains and the losses of the foreground phenotype. As coincident changes, we considered the phylogenetic branches where both the foreground amino acid and the foreground phenotype were gained, or both were lost. This approach is referred to as "GWAS" (fig. 5B).

Finally, in the third approach, we assumed that any amino acid substitution constitutes a genotype change event, and any change in the phenotype counts independently of its direction. This approach is referred to as "All changes" (fig. 5C).

These approaches correspond to different assumptions regarding the genotype-phenotype association. The "Convergence" approach assumes that the gains of the trait are associated with a gain of a specific amino acid variant, while its losses can proceed through multiple means. The "GWAS" approach assumes that both the gains and the losses of the trait are associated respectively with gains and losses of a specific amino acid variant. Finally, the "All changes" approach assumes that both the gains and the losses of the trait are associated with any changes in the encoded amino acid.

\section{Change of Site-Specific Amino Acid Propensities}

To get an alternative view of amino acid changes associated with convergent phenotype characteristics, we asked, for each amino acid site, if changes in amino acid propensities correlated with phenotype change. Among many methods for assessment of position-specific amino acid profiles, we chose the Profile Change method (Rey et al. 2018) as it was developed for studies of parallel evolution. This method assigns two amino acid profiles to each site, one for foreground and one for background branches. It then estimates differences between these two profiles in a Bayesian framework and reports the posterior probability that amino acid preferences differ between the two classes of branches. As branch classes, we used the ones for which the corresponding phenotype state was reconstructed.

\section{D Structure}

To estimate the functional role of candidate mutations, we reconstructed the 3D structure of genes that carry them. Sequences of Gallus gallus genes ND1, ND2, ND4, ND5, and ND6 were aligned with homologous genes of Ovis aries. The protein 3D structure based on homology with Ovis aries respiratory complex I (PDB:5LNK) was reconstructed by Modeller software (Sali and Blundell 1993; Fiser et al 2000; Marti-Renom et al. 2000; Webb and Sali 2016).

\section{Supplementary Material}

Supplementary data are available at Genome Biology and Evolution online.

\section{Acknowledgments}

This work was funded by the Russian Science Foundation (grant 21-74-20160 to G.A.B.).

\section{Data Availability}

Alignments are available at Dryad repository: https://datadryad.org/stash/share/gm44rbQ081b8j7Y7weOSPIVnmSvn Vje39VdAFziSNxU.

\section{Literature cited}

Armstrong AF, et al. 2008. Dynamic changes in the mitochondrial electron transport chain underpinning cold acclimation of leaf respiration. Plant Cell Environ. 31(8):1156-1169.

Capella-Gutierrez S, Silla-Martinez JM, Gabaldon T. 2009. TrimAl: tool for automated alignment trimming in large-scale phylogenetic analyses. Bioinformatics 25(15):1972-1973.

Collins C, Didelot X. 2018. A phylogenetic method to perform genomewide association studies in microbes that accounts for population structure and recombination. PLoS Comput Biol. 14(2):e1005958.

Consuegra S, et al. 2015. Patterns of natural selection acting on the mitochondrial genome of a locally adapted fish species. Genet Sel Evol. 47:58.

da Fonseca RR, Johnson WE, O'Brien SJ, Ramos MJ, Antunes A. 2008. The adaptive evolution of the mammalian mitochondrial genome. BMC Genomics. 9:119.

Das J. 2006. The role of mitochondrial respiration in physiological and evolutionary adaptation. Bioessays 28(9):890-901.

Fiedorczuk K, Letts J, Degliesposti G, et al. 2016. Atomic structure of the entire mammalian mitochondrial complex I. Nature 538(7625):406-410.

Fiser A, Do RK, Sali A. 2000. Modeling of loops in protein structures. Protein Sci. 9(9): 1753-1773.

Foote $A D$, et al. 2015. Convergent evolution of the genomes of marine mammals. Nat Genet. 47(3):272-275.

Jarvis ED, et al. 2014. Whole-genome analyses resolve early branches in the tree of life of modern birds. Science. 346(6215):1320-1331.

Ji F, et al. 2012. Mitochondrial DNA variant associated with Leber hereditary optic neuropathy and high-altitude Tibetans. Proc Natl Acad Sci U S A. 1097(19): 391-7396.

Kang L, et al. 2013. MtDNA lineage expansions in Sherpa population suggest adaptive evolution in Tibetan highlands. Mol Biol Evol. (12):302579-2587.

Kimball RT, et al. 2019. A phylogenomic supertree of birds. Diversity 11(7):109.

Kosakovsky Pond SL, Frost SDW, Muse SV. 2005. HyPhy: hypothesis testing using phylogenies. Bioinformatics 21(5):676-679.

Lim MCW, Witt CC, Graham CH, Dávalos LM. 2019. Parallel Molecular Evolution in Pathways, Genes, and Sites in High-Elevation 
Hummingbirds Revealed by Comparative Transcriptomics. Genome Biol Evol. 11(6):1573-1585.

Lucassen M, Koschnick N, Eckerle LG, Pörtner HO. 2006. Mitochondrial mechanisms of cold adaptation in cod (Gadus morhua L.) populations from different climatic zones. J Exp Biol. 209(Pt 13):2462-2471.

Marti-Renom MA, et al. 2000. Comparative protein structure modeling of genes and genomes. Annu Rev Biophys Biomol Struct. 29:291-325.

Minh BQ, et al. 2020. IQ-TREE 2: New models and efficient methods for phylogenetic inference in the genomic era. Mol Biol Evol. (5):371530-1534.

Natarajan C, et al. 2016. Predictable convergence in hemoglobin function has unpredictable molecular underpinnings. Science 354(6310):336-339.

Parker J, et al. 2013. Genome-wide signatures of convergent evolution in echolocating mammals. Nature 502(7470): 228-231.

Ranwez V, Harispe S, Delsuc F, Douzery EJP. 2011. MACSE: Multiple Alignment of Coding SEquences accounting for frameshifts and stop codons. PLoS One 6(9):e22594.

Rey C, Guéguen L, Sémon M, Boussau B. 2018. Accurate detection of convergent amino-acid evolution with PCOC. Mol Biol Evol. 35(9):2296-2306.

Sali A, Blundell TL. 1993. Comparative protein modelling by satisfaction of spatial restraints. J Mol Biol. (3):234779-815.

Scott GR, et al. 2011. Molecular evolution of cytochrome c oxidase underlies high-altitude adaptation in the bar-headed goose. Mol Biol Evol. 28(1):351-363.

Shen YY, et al. 2010. Adaptive evolution of energy metabolism genes and the origin of flight in bats. Proc Natl Acad Sci U S A. 107(19):8666-8671
Silva G, Lima FP, Martel P, Castilho R. 2014. Thermal adaptation and clinal mitochondrial DNA variation of European anchovy. Proc R Soc B. 281(1792):20141093.

Thomas GWC, Hahn MW. 2015. Determining the null model for detecting adaptive convergence from genomic data: a case study using echolocating mammals. Mol Biol Evol. 32(5):1232-1236.

Toews DPL, Mandic M, Richards JG, Irwin DE. 2014. Migration, mitochondria, and the yellow-rumped warbler. Evolution. (1):68241-255.

Tomasco IH, Lessa EP. 2011. The evolution of mitochondrial genomes in subterranean caviomorph rodents: adaptation against a background of purifying selection. Mol Phylogenet Evol. 61(1):64-70.

Webb B, Sali A. 2016. Comparative protein structure modeling using modeller. Curr Protoc Bioinformatics. 54:5.6.1-5.6.37.

Xu SQ, et al. 2005. A mitochondrial genome sequence of the Tibetan antelope (Pantholops hodgsonii), Genomics Proteomics Bioinformatics. 3(1):5-17.

Yuan M-L, et al. 2018. Mitochondrial phylogeny, divergence history and high-altitude adaptation of grassland caterpillars (Lepidoptera: Lymantriinae: Gynaephora) inhabiting the Tibetan Plateau. Mol Phylogenet Evol. 122:116-124.

Zhang HX, et al. 2013. Mitochondrial genome sequences of Artemia tibetiana and Artemia urmiana: assessing molecular changes for high plateau adaptation. Sci China Life Sci. 56(5): 440-452.

Zhou T, Shen X, Irwin DM, Shen Y, Zhang Y. 2014. Mitogenomic analyses propose positive selection in mitochondrial genes for high-altitude adaptation in galliform birds. Mitochondrion 18:70-75.

Zou Z, Zhang J. 2015. No genome-wide protein sequence convergence for echolocation. Mol Biol Evol. 32(5):1237-1241.

Associate editor: Jay Storz 